\title{
Review
}

\section{Effect of the Timing of Restoration on Implant Marginal Bone Loss: A Systematic Review}

\author{
Fernando Suarez, * Hsun-Liang Chan, * Alberto Monje, * Pablo Galindo-Moreno, ${ }^{\dagger}$ \\ and Hom-Lay Wang*
}

Background: The advancement in implant dentistry has allowed shortened treatment time by restoring the implants earlier. Whether the timing of restoration has an impact on implant marginal bone level has not been systematically analyzed. The aim of this study is to compare marginal bone loss (MBL) between implants that were restored with the following protocols: 1) immediate restoration/loading (IR/L); 2) early loading (EL); and 3) conventional loading (CL).

Methods: An electronic literature search from three databases (until November 2011) and a hand search in implantrelated journals were conducted. Clinical human studies in English language that had reported a comparison of MBL between implants with IR/L, EL, or CL with $\geq 12$-month follow-up were included. In addition, the minimal number of implants had to be 10 for each group. Implants with both immediate placement (IP) and delayed placement (DP) were included and analyzed separately. An assessment of the publication bias for the included randomized clinical trials (RCTs) was performed.

Results: The initial search resulted in 1,640 articles, of which 27 articles in full text were further evaluated for eligibility. Finally, 11 studies (eight RCTs, two controlled clinical trials, and one retrospective study) were qualified and classified into four groups: 1$) I R / L+D P$ versus $C L+D P(n=6$ articles); 2) $I R+D P$ versus $E L+D P(n=2$ articles $) ; 3) E L+D P$ versus $C L+D P(n=1$ article); and (4) IL + IP versus CL + IP ( $n=2$ articles). A meta-analysis performed for group 1 showed 0.09 $\mathrm{mm}$ (95\% confidence interval $=-0.27$ to $0.09 \mathrm{~mm})$ difference in the mean MBL, favoring the IR/L protocol but without significant difference $(P=0.33)$. No significant difference in MBL was found for groups 2 through 4 after adjusting for the implant placement level. The eight RCTs were determined to be at moderate-to-high risk of publication bias.

Conclusions: This meta-analysis does not show an effect of the timing of restorations on implant MBL. The selection of restoration protocols should be based on factors other than MBL. J Periodontol 2013;84:159-169.

\section{KEY WORDS}

Alveolar bone loss; dental implantation; dental implants; dental prosthesis design; dental prosthesis, implantsupported.

\footnotetext{
* Graduate Periodontics, Department of Periodontics and Oral Medicine, University of Michigan School of Dentistry, Ann Arbor, MI

$\dagger$ Department of Oral Surgery, University of Granada, Granada, Spain.
}

$\tau$ mplant dentistry has evolved to a stage that a high implant survival rate alone, achieved by the conventional loading (CL) approach, could no longer satisfy the patients and health care providers. Long waiting time for the implant to be osseointegrated before the restoration can be placed discourages patient acceptance of implant therapy. The restoration of chewing and phonetic function and esthetics that implants can provide is delayed. Different loading protocols have thus been developed and subsequently classified. ${ }^{1,2}$ According to the 2004 consensus statements, ${ }^{1}$ immediate restoration (IR) refers to the insertion of a restoration within 48 hours of implant placement but not in occlusion with the opposing dentition, whereas immediate loading (IL) is to place the restoration in occlusion with the opposing dentition within 48 hours of implant placement. Early loading (EL) is defined when a prosthesis is placed $\geq 48$ hours after the implant placement but not later than 3 months afterward.

The rationale for the $\mathrm{CL}$ protocol was to keep the implant in an undisturbed environment during the healing period. ${ }^{3}$ It was believed that applying forces to the implant during that critical period might cause micromovement at the implantbone surface, which in turn results in fibrous encapsulation and eventually implant failure. Understanding the process of how osseointegration is achieved and

doi: 10.1902/jop.2012.120099 
the advances in implant designs have allowed a faster loading protocol. ${ }^{4}$ The primary stability that is achieved by mechanical locking of the implant to the bone serves to prevent the occurrence of micromovement, even when the implant is subject to occlusal loads. When the primary stability starts to decrease, the secondary stability catches up and takes over to resist occlusal forces. Certainly, methods to decrease the loading, such as splinting, increasing the number of implants, and eliminating lateral contacts, have been applied to optimize the loading condition. As long as the forces do not violate the orchestrated implant healing process, it is possible to restore or load the implant immediately.

EL protocol has been validated for full-arch rehabilitations with various levels of evidence in a review article. ${ }^{5}$ Briefly, EL of overdentures or fixed dentures in either jaw is clinically documented or clinically well documented. IL is clinically well documented for fixed dentures in either jaw and for overdentures in the mandible. However, insufficient scientific or clinical documentation was found for IL of maxillary overdentures. Included in the documentation within the same review article, ${ }^{5}$ implants with IL or EL protocol had a survival rate $(87.2 \%$ to $100 \%)$ comparable to those with CL protocol. High survival rate (average of $96.4 \%$ to $98.2 \%$ ) was also found for early and IR/L implants for single-tooth and partial-arch applications, although most articles were case reports. ${ }^{6}$ It is important to note that some important factors, such as bone quality and quantity, implant design, splinting of implants, and prosthetic design, have to be considered for achieving predictable outcomes. ${ }^{7}$

To further shorten treatment time and maintain soft-tissue architecture, immediate implant placement in fresh sockets and provisionalization has been advocated, especially for the restoration of the esthetic zone. ${ }^{8,9}$ With this technique, less tissue damage might be expected than the standard protocol because only one surgery is required. A systematic review ${ }^{10}$ showed that this procedure is predictable for single-tooth replacement in the anterior maxilla, with certain precautions being considered.

Other than the presence of implants in situ, MBL with time is another criterion to assess implant success. It can be classified into early and late MBL. Late MBL is most likely related to peri-implantitis. ${ }^{11}$ The causes of early bone loss are less understood and have been hypothesized, including the following: 1) surgical trauma; 2) the presence of the microgap between the fixture and the abutment; 3 ) the remodeling process to restore biologic width; and 4) occlusal overloading. ${ }^{12}$ It has been shown that the occlusal stresses primarily concentrated at the crestal bone. Bone remodeling is a function of stresses and a net result from bone apposition and resorption. ${ }^{13}$ Excessive loading may cause progressive marginal bone loss, ${ }^{14-17}$ which might be more likely to occur when the bond between the implant and bone has not been established during the initial healing phase. In light of the fact that occlusal loading has a role on bone remodeling, it is the aim of this meta-analysis to evaluate the effect of the timing of restoration on implant MBL.

\section{MATERIALS AND METHODS}

A search of three electronic databases, including PubMed, Ovid (MEDLINE), and Cochrane Central, for relevant studies published in English was performed in November 2011 by two examiners (FS and $\mathrm{H}$-LC). There was no limit to the time of publication; however, only articles in English language were considered. The search terms used, in which mh represented the MeSH term and tiab represented the title or abstract, were as follows: "Jaw, edentulous" [mh] OR "Alveolar process"[mh] OR "Alveolar bone loss" [mh] OR “Dental implantation" [mh] OR "Dental implants" [mh] OR "Dental prosthesis design" [mh] OR "Denture" [mh] OR "Dental prosthesis, implant-supported" [mh] OR "Healed socket"[tiab] OR "Fresh socket"[tiab] AND ("Immediate"[tiab] OR "Early"[tiab] OR "Delayed"[tiab] OR "Immediate non-occlusal"[tiab] OR "Functional"[tiab] OR "Non-functional"[tiab]) AND ("Provisionalization" [tiab] OR "Restoration"[tiab] OR "Loading"[tiab]).

In addition, a search for references in the included papers was performed. Finally, a hand search (January 2000 through November 2011) was performed in dental journals, including Journal of Oral and Maxillofacial Implants; Clinical Implant Dentistry, and Related Research; Clinical Oral Implants Research; Implant Dentistry; European Journal of Oral Implantology;' Journal of Oral Implantology; International Journal of Oral and Maxillofacial Surgery; Journal of Oral and Maxillofacial Surgery; Journal of Dental Research; International Journal of Prosthodontics; Journal of Prosthetic Dentistry; Journal of Clinical Periodontology; Journal of Periodontology; and The International Journal of Periodontics and Restorative Dentistry.

Articles were included if the following criteria were fulfilled: 1) human clinical trials that compared marginal bone loss between implants with immediate, early, or delayed loading/restoration and $\geq 10 \mathrm{im}$ plants in each group with $\geq 12$-month follow-up. The implants could be placed in either healed or fresh sockets but had to be restored with fixed prostheses. Articles were excluded if they fell into one of the following categories: case reports/series, review articles, or clinical trials with $<10$ implants with insufficient follow-up time or with implants that were placed along with bone grafts or restored with 
PubMed, Ovid (MEDLINE), and

Cochrane Central databases searched

Limits: English-language articles only

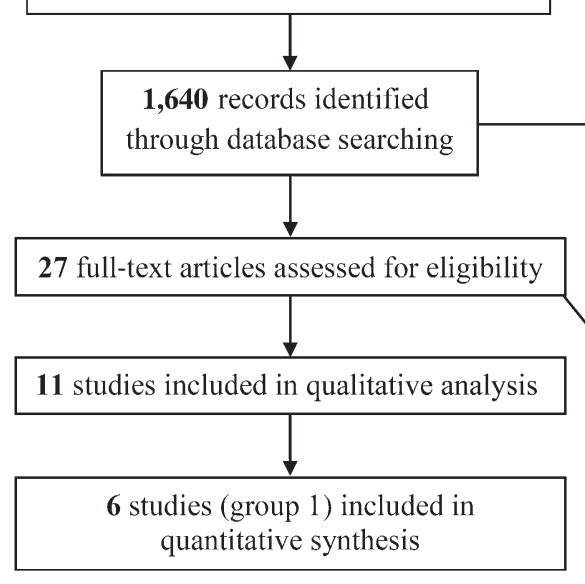

1,613 records excluded based on exclusion/inclusion criteria

16 full-text articles excluded: Insufficient data on marginal bone loss $(\mathrm{n}=11)$ Evaluated placement protocols only $(\mathrm{n}=1)$ Insufficient follow-up $(\mathrm{n}=1)$ Used bone grafting materials $(n=1)$ Implants restored with overdentures $(n=2)$

Figure I.

Flowchart of the screening process.

removable overdentures. Articles with different implant systems or with different implant placement protocols (e.g., healed versus extraction sockets) between experimental groups were also excluded. Potential articles were independently reviewed in full text by two examiners (FS and H-LC). The final decision on the included articles was made by mutual agreement of the two examiners.

\section{Data Analyses}

The outcome was implant MBL as assessed radiographically. Data including implant numbers and mean value and standard deviation of marginal bone loss at 1-year follow-up were extracted from each included article and transported to a commercially available software package ${ }^{\ddagger}$ for the meta-analysis. The contributions of each article to the primary outcome were weighed based on the sample size, and the random-effect model was chosen. Publication bias was examined by the funnel plot in which the standard error of the difference in mean marginal bone loss was plotted against the difference in mean from the included articles. The reporting of this metaanalysis adhered to the Preferred Reporting Items for Systematic Review and MetaAnalyses statement. ${ }^{18}$

\section{Risk of Bias Assessment}

The criteria used for assessment of the risk of bias for the selected randomized controlled trials were derived from the checklist of the Cochrane Center ${ }^{19}$ and the CONSORT (Consolidated Standards of Reporting Trials) statement, ${ }^{20}$ including the following: 1 ) representative of general population; 2) defined inclusions/ exclusions; 3) randomization methods; 4) allocation concealment method; 5) mask of the examiner; 6 ) intervention different only; and 7) patient drop-out and analysis accounts for patient losses. One of three categories were given after the assessment: 1) low risk of bias if no or one criterion was missing; 2) moderate risk of bias when two criteria were missing; and 3) high potential risk of bias if $\geq 3$ criteria were missing. Two reviewers (FS and H-LC) assessed the risk of publication bias for the included randomized clinical trials.

\section{RESULTS}

The screening process was represented in Figure 1. The initial screening yielded a total of 1,640 articles. After initial screening of their titles and abstracts, 27 articles were further evaluated in full text, of which 11 articles $^{21-31}$ were selected for this systematic review. Interexaminer agreement in selecting articles was 0.9. The reasons for exclusion after full-text evaluation included the following: 1) no report on $\mathrm{MBL}^{32-39} 2$ ) investigating the effect of the timing of implant placement; ${ }^{40} 3$ ) MBL reported as a range only; ${ }^{41-43} 4$ ) insufficient follow-up; ${ }^{44}$ ) the use of grafting materials; ${ }^{45}$ and 6 ) the use of overdentures as final restorations. ${ }^{46,47}$

\section{Characteristics of the Included Articles}

Study design and length of the follow-up. The 11 articles were subcategorized into four groups based on their loading and placement protocols: Group 1: IR/L versus $C L$ implants, in which the implants were placed in healed ridges (six articles); Group 2: IR versus EL implants, in which the implants were placed in healed ridges (two articles); Group 3: ER versus CL implants, in which the implants were placed in healed ridges (one article); and Group 4: IL versus CL implants, in which the implants were placed in fresh sockets (two articles).

Table 1 summarizes the characteristics of the included studies. A meta-analysis was performed for group 1 , whereas a summary of the main outcome was provided for groups 2 through 4 .

Eight articles $22-24,26-28,30,31$ were classified as a randomized controlled trial, two ${ }^{25,29}$ were controlled clinical trials, and the other one ${ }^{21}$ was a retrospective study.

Implant sample size. The total number of test and control implants that were available for data analysis

\footnotetext{
‡ CMA, Biostat, Englewood, NJ.
} 


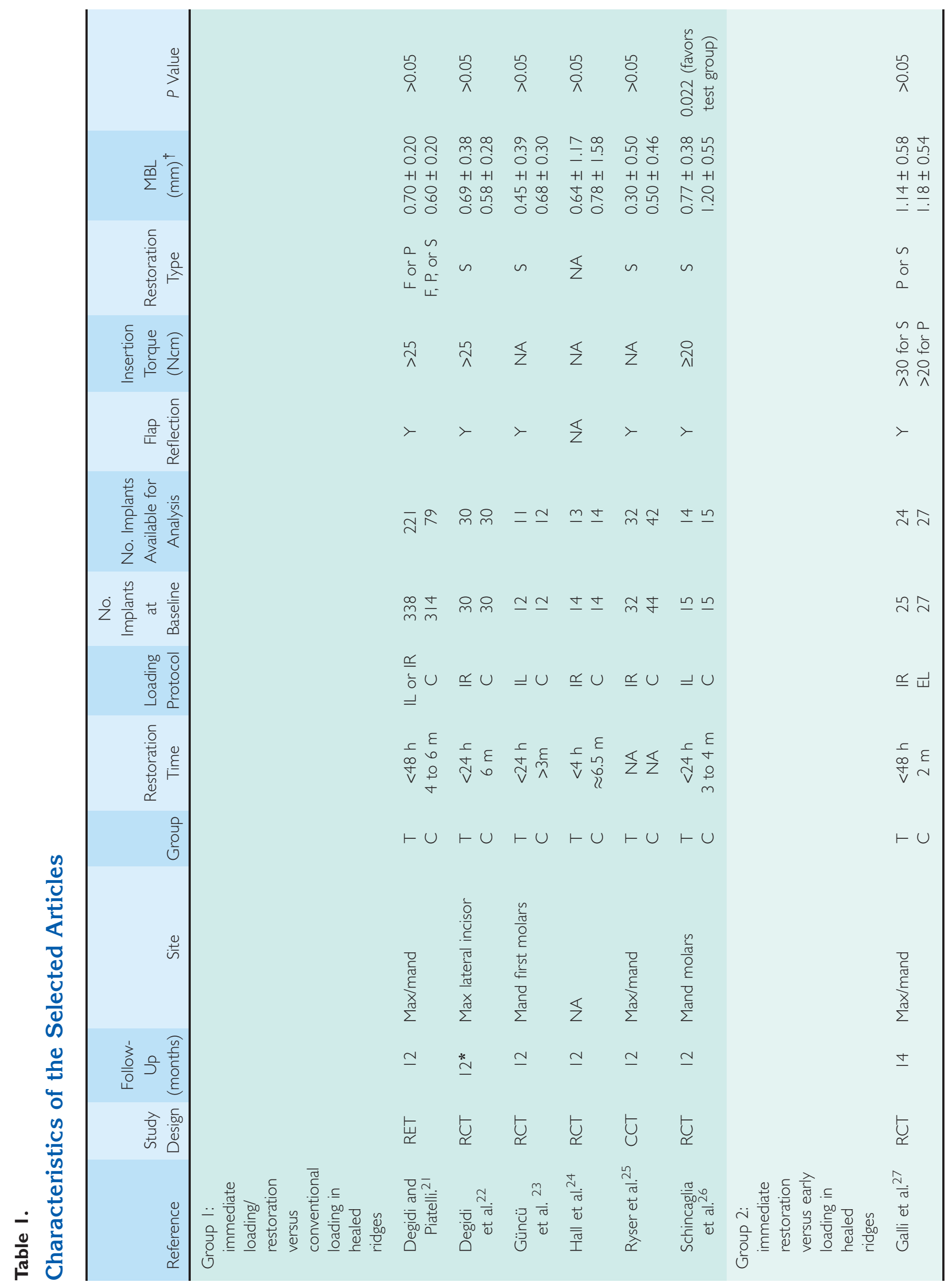




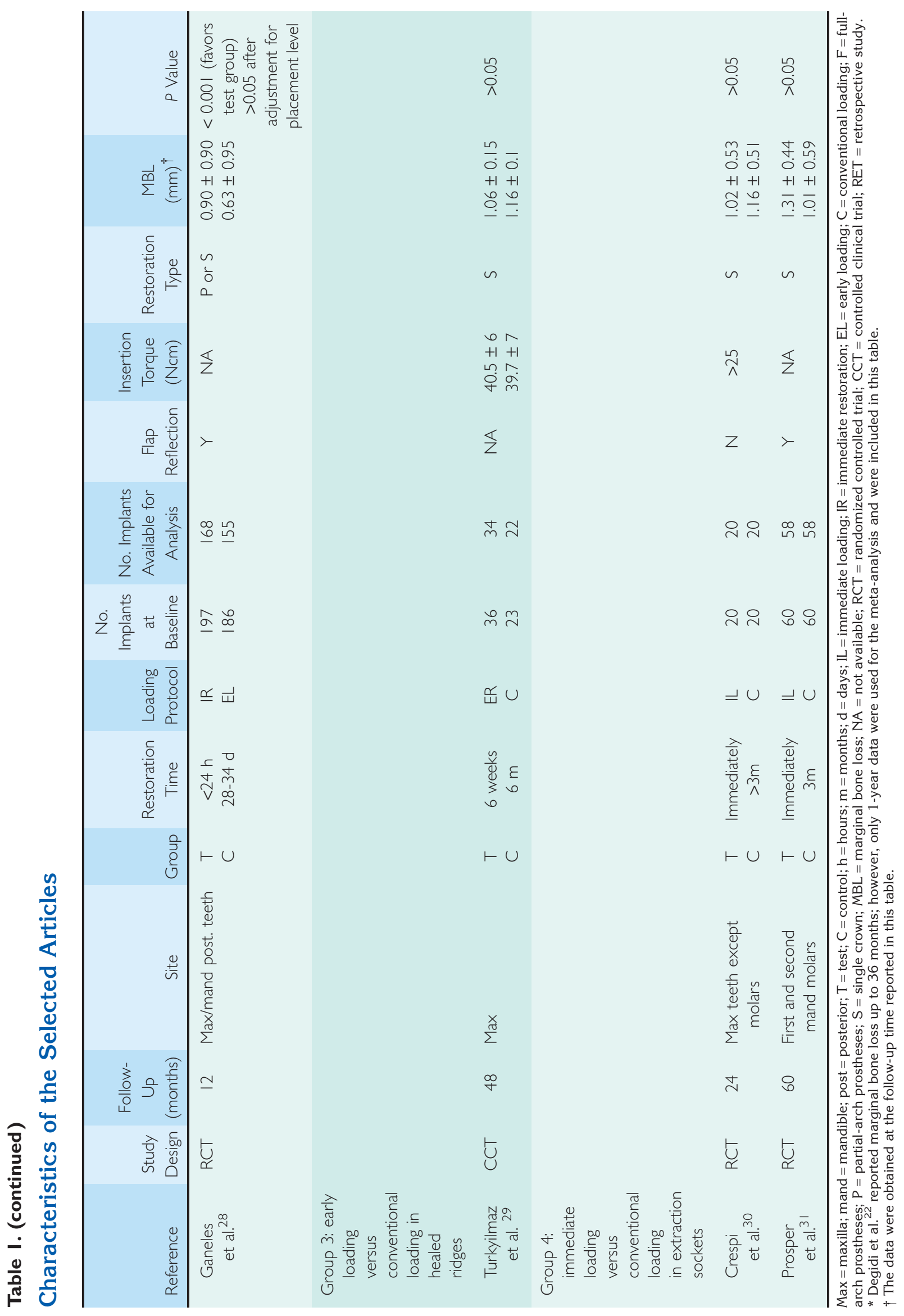


was 310 and 182 in group 1, 192 and 182 in group 2, 34 and 22 in group 3, and 80 and 80 in group 4 . Of the 11 articles, a multicenter study ${ }^{28}$ provided the largest sample size, with 168 immediately loaded and 155 early restored implants, whereas the study with the smallest sample size ${ }^{23}$ had 11 and 12 immediately and delayed restored implants, respectively.

Implant location, restoration type, and protocol and surgical specifications. Group 1: Two studies ${ }^{21,25}$ did not restrict the type of teeth to be restored. One ${ }^{22}$ only studied maxillary lateral incisors; two ${ }^{23,26}$ only studied mandibular molars. One study ${ }^{24}$ did not mention the type of tooth studied. One study ${ }^{21}$ included fully and partially edentulous ridges; four studies $^{22,23,25,26}$ included single missing teeth. Most immediately restored implants did not contact in centric occlusion during temporization, ${ }^{22,24,25}$ except those replacing fully edentulous ridges ${ }^{21}$ and single mandibular molars. ${ }^{23,26}$ Three studies ${ }^{21,22,26}$ specified the minimal insertion torque values ( $\geq 20$ or $>25 \mathrm{Ncm}$ ) that allowed for immediate restoration/loading procedures. In five studies, ${ }^{21-23,25,26}$ mucoperiosteal flaps were raised during the implant surgery, whereas the other ${ }^{24}$ one did not mention the type of surgical method.

Group 2: In both studies, ${ }^{27,28}$ the test implants were immediately restored within 48 hours without occlusal contacts, whereas the control implants were loaded on days 28 through $34^{28}$ or at month $2 .^{27}$ Implants were placed in partially edentulous ridges or single-tooth gaps in both studies. All tooth types were studied in one article, ${ }^{27}$ whereas only posterior teeth in both jaws were studied in another article. ${ }^{28}$ One study ${ }^{27}$ specified that the minimal insertion torque values for immediate restoration procedures were $>20$ or $>30 \mathrm{Ncm}$ in partially edentulous and single-tooth restorations, respectively. Mucoperiosteal flaps were raised in both studies.

Group 3: One study ${ }^{29}$ was available for data extraction. Implants in the test group were restored at 6 weeks after implant placement, whereas the control group implants were restored 6 months after the surgery. Only single implants were studied. All implants were placed in the maxilla, replacing all types of teeth.

Group 4: Single-tooth implants located in the maxilla except molar sites ${ }^{30}$ or in the mandibular molar sites ${ }^{31}$ were studied. The test implants were loaded immediately, whereas the controls were loaded 3 months after. Mucoperiosteal flaps were raised in one study ${ }^{31}$ but not the other one. ${ }^{30}$ Minimal insertion torque $(>25 \mathrm{Ncm})$ was required for immediately loaded implants. ${ }^{30}$

\section{RESULTS}

\section{Group 1}

The meta-analysis showed that the weighted mean difference in marginal bone loss among implants that were loaded or restored immediately and those that were conventionally loaded was $-0.09 \mathrm{~mm}(95 \%$ confidence interval $=-0.27$ to $0.09 \mathrm{~mm}$ ), favoring the test group but without statistical significance $(P=$ 0.33) (Fig. 2). The funnel plot showed asymmetric distributions, indicating the possibility of publication bias (Fig. 3). Because of the fact that most articles showed no significant differences, the results of the funnel plot might be negligible.

\section{Groups 2 through 4}

Figure 4 summarizes the comparison of the marginal bone loss for groups 2 and 4 . Two articles ${ }^{27,28}$ were available for comparisons between IR and EL implants. In one multicenter, randomized controlled study, ${ }^{27} 14$ months after implant surgery, the mean marginal bone loss was not different $(1.14 \pm 0.58$ and $1.18 \pm 0.54 \mathrm{~mm}$ for the immediate and early restoration group, respectively). In the other multicenter study, ${ }^{28}$ implants that were immediately restored lost significantly more marginal bone than early loaded ones $(0.90 \pm 0.90$ versus $0.63 \pm 0.95 \mathrm{~mm})$. However, after adjusting for the differences in implant position in relation to marginal bone level, such differences did not exist anymore. When comparing MBL between implants that were early and conventionally loaded in healed ridges, no differences ( $1.06 \pm 0.15$ versus $1.16 \pm 0.1$, respectively) were found 4 years after implant surgery. ${ }^{29}$ For implants that were placed in fresh sockets, whether they were loaded immediately or conventionally, the bone level was not significantly different. 30,31

\section{Results of Risk of Bias Assessment}

The eight included randomized controlled trials ${ }^{22-24,26-28,30,31}$ were categorized into either moderate or high risk of bias (Table 2). The potential bias originated from the following: 1) no specification of the inclusion/exclusion criteria 24 ; 2) no mention of randomization methods $27,30,31$; 3) no mention of allocation concealment method 22,26,27,30,31; 4) no masking of the examiners ${ }^{24,30}$; and 4) no analysis accounting for patient losses. ${ }^{24}$

\section{DISCUSSION}

This study suggests that the timing of restoration does not influence MBL around implants at short-term follow-up. Evidence on the long-term effect is limited, although it is less likely a difference will be seen. For a successful implant, most MBL occurred during the first year of function, after which the loss is negligible. Additionally, once the osseointegration is achieved and permanent restorations are placed, the differences between various loading protocols do not exist anymore. One study ${ }^{22}$ was available for 3-year followup and did not show difference in MBL between immediately and conventionally loaded implants (0.85 


\begin{tabular}{|c|c|c|c|}
\hline \multirow[t]{2}{*}{ Study Name } & \multicolumn{2}{|c|}{ Sample Size } & \multirow[b]{2}{*}{$\begin{array}{c}\text { Difference } \\
\text { in Means }\end{array}$} \\
\hline & $\begin{array}{l}\text { Immed. } \\
\text { Immed/Rest. }\end{array}$ & $\begin{array}{l}\text { Conv. } \\
\text { Load }\end{array}$ & \\
\hline Degidi \& Piatelli ${ }^{21}$ & 221 & 79 & 0.10 \\
\hline Degidi et al. ${ }^{22}$ & 30 & 30 & 0.11 \\
\hline Güncü et al. ${ }^{23}$ & 11 & 12 & -0.23 \\
\hline Hall et al. ${ }^{24}$ & 13 & 14 & -0.14 \\
\hline Ryser et al. ${ }^{25}$ & 32 & 42 & -0.20 \\
\hline Schincaglia et al. ${ }^{26}$ & 14 & 15 & -0.43 \\
\hline Total & 321 & 192 & -0.09 \\
\hline
\end{tabular}

\begin{tabular}{crrr}
\multicolumn{4}{c}{ Statistics for Each Study } \\
$\begin{array}{c}\text { Standard } \\
\text { Error }\end{array}$ & $\begin{array}{r}\text { Lower } \\
\text { Limit }\end{array}$ & $\begin{array}{r}\text { Upper } \\
\text { Limit }\end{array}$ & $\boldsymbol{P}$ Value \\
0.03 & 0.04 & 0.16 & 0.00 \\
0.09 & -0.07 & 0.29 & 0.22 \\
0.15 & -0.52 & 0.06 & 0.13 \\
0.53 & -1.18 & 0.90 & 0.79 \\
0.11 & -0.42 & 0.02 & 0.07 \\
0.17 & -0.76 & -0.10 & 0.01 \\
0.09 & -0.27 & 0.09 & 0.33
\end{tabular}

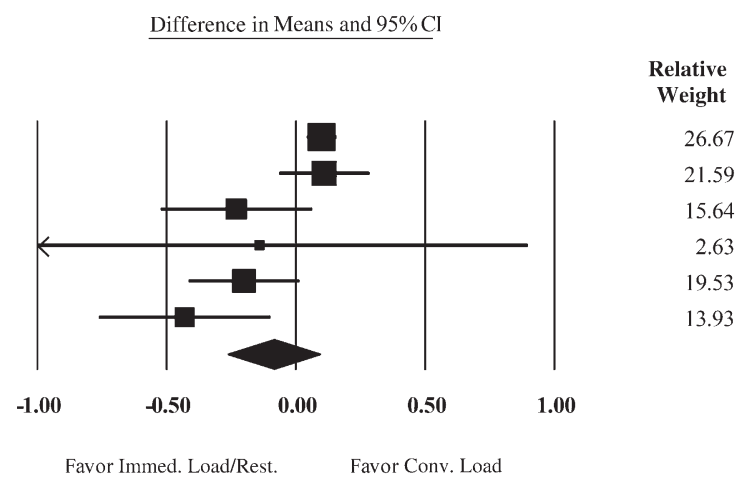

Figure 2.

A meta-analysis for the comparison of marginal bone loss for group 1 . The weighted mean difference in marginal bone loss between implants with immediate and delayed restorations was $-0.09 \mathrm{~mm}(95 \%$ confidence interval $=-0.27$ to $0.09 \mathrm{~mm}$ ), favoring immediate restoration but without statistical significance $(\mathrm{P}=0.33)$.

\pm 0.71 versus $0.75 \pm 0.63 \mathrm{~mm})$. Another ${ }^{31}$ provided 6 -year data, which concluded that MBL of immediately placed and restored implants was comparable to that of immediately placed and conventionally loaded implants.

Implant insertion torque has to be considered for the application of IR/L and EL protocol because it is related to primary stability and implant survival. An animal study ${ }^{48}$ showed that high insertion torque increased implant primary stability. Implant failure rate was reduced by $20 \%$ when every $9.8 \mathrm{Ncm}$ was added to the insertion torque. ${ }^{49}$ An adequate torque value for immediate and early loaded implants has not been established yet. One study ${ }^{49}$ suggested that, to achieve osseointegration, $\geq 32 \mathrm{Ncm}$ is necessary, whereas a recent clinical trial ${ }^{50}$ showed that $25 \mathrm{Ncm}$ seemed more than sufficient to yield a favorable clinical outcome. Most studies included in the present review suggested that the insertion torque of $\geq 20 \mathrm{Ncm}$ had to be achieved before the implants could be immediately restored or loaded. Modifications of surgical procedures, e.g., undersized site preparation, have shown to enhance primary stability and should be considered for immediately loaded implants. ${ }^{51}$

In one study, ${ }^{26}$ single implants replacing the mandibular molars were immediately loaded and showed significantly less MBL It is possible that occlusal loading might help maintain marginal bone level. Bone remodels in reaction to the stresses that are applied to it. ${ }^{13}$ Additionally, whether the remodeling is anabolic or catabolic depends on the magnitude of forces. Excessive forces cause microfracture and eventual bone resorption; conversely, optimal forces can stimulate bone apposition. At molecular levels, bone cells are capable of sensing mechanical stimuli, resulting in bone mass and morphology changes as an adaptive response. ${ }^{52}$ Almost all intracellular trans-

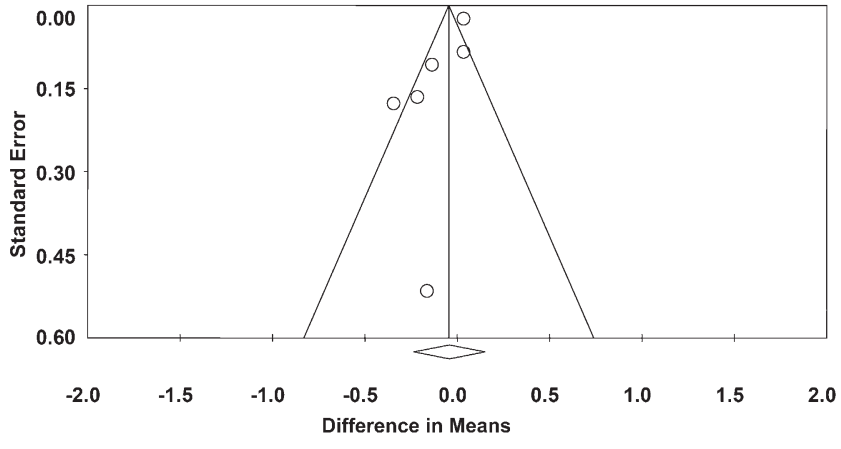

Figure 3.

The funnel plot for the meta-analysis showed asymmetric distributions, indicating the possibility of publication bias. Nevertheless, because of the fact that most papers showed no statistical differences, the results of the funnel plot might be negligible.

duction signal cascades are activated in this response, from intracellular cyclic adenosine monophosphate, inositol trisphosphate, calcium guanidine regulatory proteins, to mitogen-activated protein kinase. The end results are the recruitment of either osteoblasts or osteoclasts. Animal studies have shown higher percentage of bone-implant contacts, ${ }^{53,54}$ higher peri-implant bone density, ${ }^{53,55}$ and the presence of transversely oriented collagen fibers in the periimplant bone ${ }^{56}$ for immediately loaded implants. More clinical studies are needed to test the beneficial role of IL on maintaining marginal bone.

Immediate implant placement and provisionalization is technically more challenging but can be predictable as long as certain criteria are met. A systematic review ${ }^{10}$ reported $100 \%$ short-term implant survival rate in almost all included articles. This approach might also help preserve peri-implant hard and soft tissue. Case series investigating 

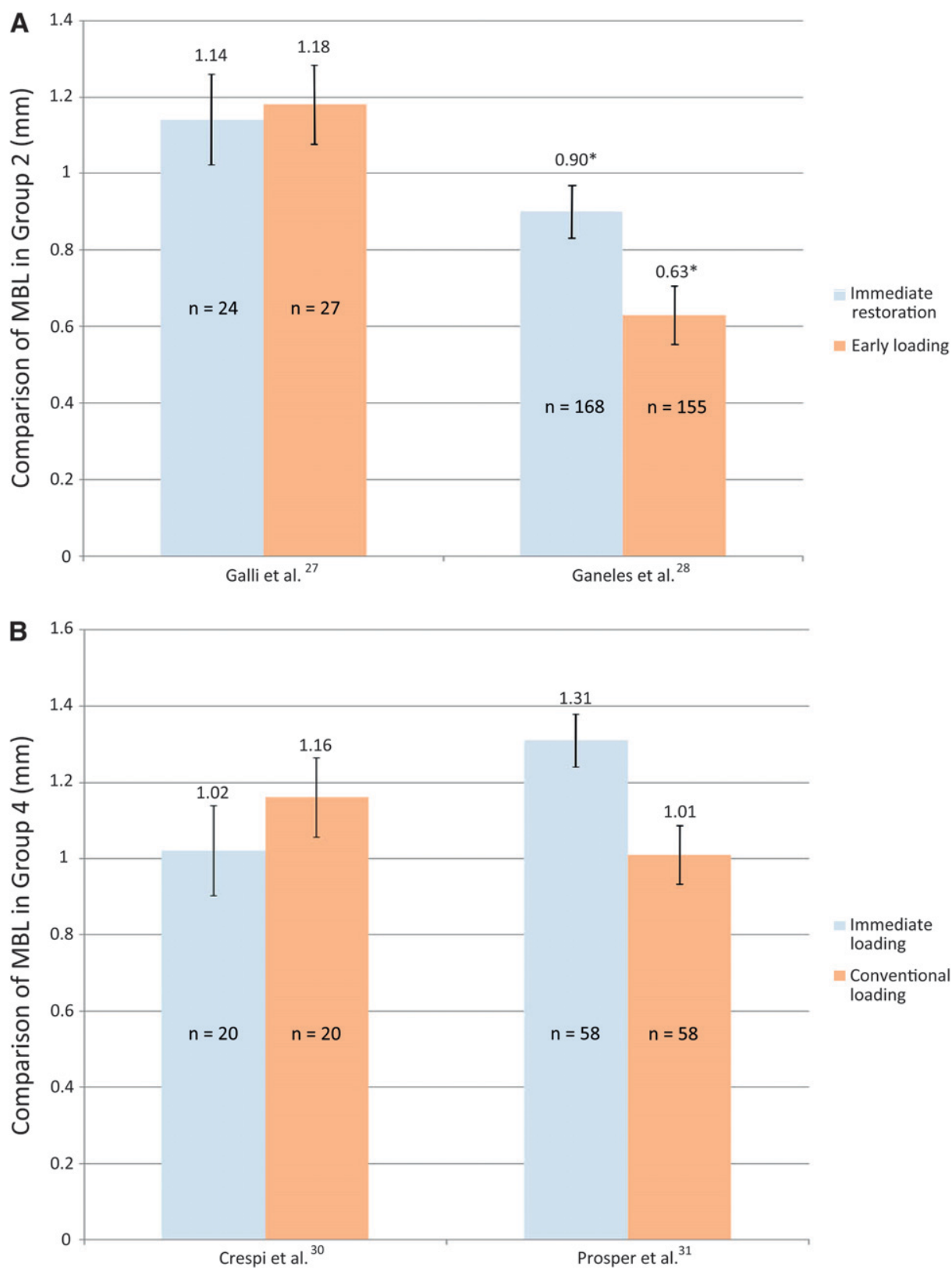

Figure 4.

Bar charts summarizing the comparison ofMBL in group 2 (A) and group 4 (B). A significant difference was found by Ganeles and Wismeijer (as marked with *). According to the original article, after adjusting for implant placement level, the difference did not exist anymore.

implants that were placed and restored immediately in the esthetic zone showed minimal marginal bone changes (ranging from $0.3^{57}$ to $0.67^{58}$ to $\approx 1 \mathrm{~mm}^{59}$ ). Additionally, limited loss of papilla height $(<0.5 \mathrm{~mm})$ was reported. ${ }^{60}$ There was even some papilla rebound after several years of function. ${ }^{58,59}$ The preservation of papilla height might have been the combined results of the reduced number of surgical procedures, maintenance of the bone spike of the adjacent teeth, and the contours of the temporary restorations. One concern about this approach is the recession of facial mucosa, which was progressive with time. ${ }^{58}$ Up to 1 year, the mean recession was $0.55 \mathrm{~mm}$. At the mean follow-up time of year 4 , the recession was $1.13 \mathrm{~mm}$. Gingival biotype is correlated with the amount of recession, with thick tissue biotype yielding less loss. ${ }^{58}$

A head-to-head comparison of MBL between implants after immediately placed and restored protocol and immediately placed and conventionally restored protocols is limited. The present review identified two articles ${ }^{30,31}$ that had addressed this issue, both of which did not show a difference in MBL between the two experimental groups.

Limitations of this systematic review include small sample size, only English articles as a selection criterion, and heterogeneities in the study designs of the included papers. Nonetheless, this review provides updated information on potential effects of the timing of restorations on implant marginal bone level. Future research should focus on the effect of immediate/early loading on grafted bone.

\section{CONCLUSIONS}

Eleven studies ${ }^{21-31}$ (eight randomized controlled trials, two controlled clinical trials, and one retrospective study) were qualified for the evaluation of implant MBL between different loading protocols. Nine articles $^{21-29}$ evaluated implants that were placed in healed sockets, of which six ${ }^{21-26}$ and two ${ }^{27,28}$ studies compared MBL between IL/R and CL and between IR and EL, respectively. The other study ${ }^{29}$ made the comparison EL loading and CL. Two articles ${ }^{30,31}$ investigated marginal bone level changes of implants that were placed in fresh sockets and received either immediate or delayed loading. Available evidence did not show an effect of the timing of restoration on marginal bone level. 


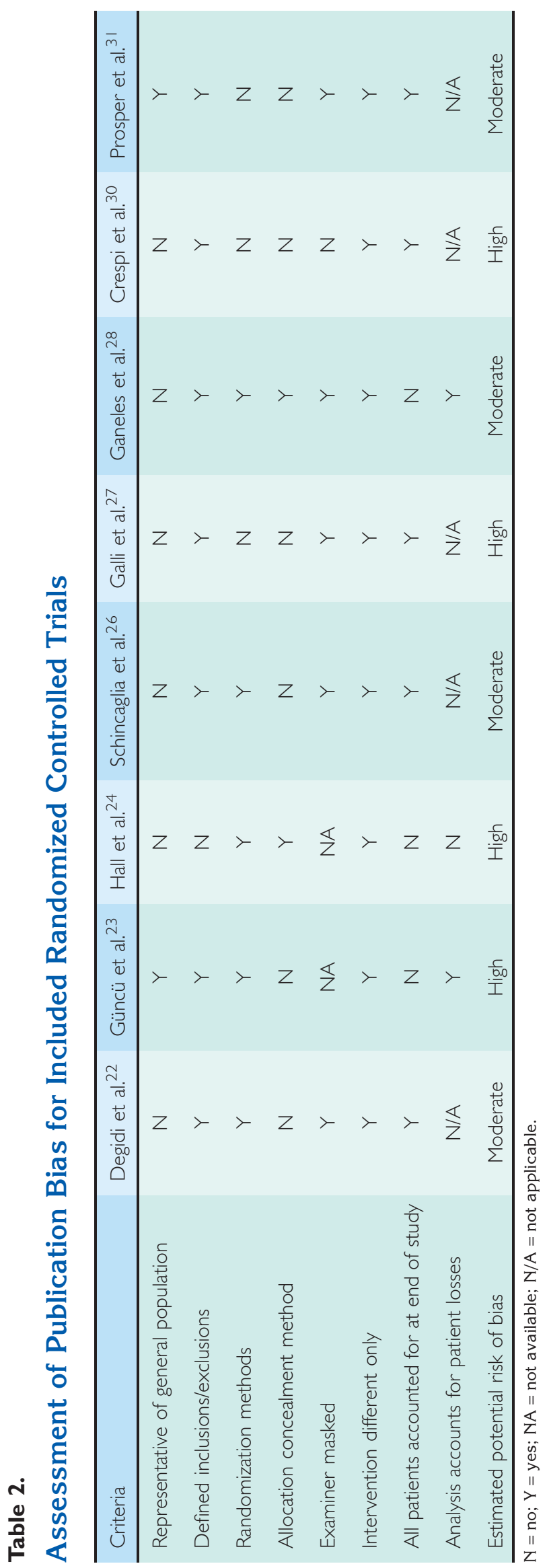

\section{ACKNOWLEDGMENTS}

This study was partially supported by the University of Michigan Periodontal Graduate Student Research Fund. The authors acknowledge Mr. Kerby A. Shedden, Associate Professor at the Department of Statistics, for his guidance in conducting metaanalyses, and Mr. Mark MacEachern, a liaison services librarian in the Taubman Health Sciences Library, for providing consultations on the literature search. Both individuals are currently affiliated with the University of Michigan, Ann Arbor, MI. The authors report no conflicts of interest related to this study.

\section{REFERENCES}

1. Cochran DL, Morton D, Weber HP. Consensus statements and recommended clinical procedures regarding loading protocols for endosseous dental implants. Int J Oral Maxillofac Implants 2004;19(Suppl.):109113.

2. Wang HL, Ormianer Z, Palti A, Perel ML, Trisi P, Sammartino G. Consensus conference on immediate loading: The single tooth and partial edentulous areas. Implant Dent 2006;15:324-333.

3. Brånemark PI, Hansson BO, Adell R, et al. Osseointegrated implants in the treatment of the edentulous jaw. Experience from a 10-year period. Scand J Plast Reconstr Surg Suppl 1977;16:1-132.

4. Szmukler-Moncler S, Piattelli A, Favero GA, Dubruille $\mathrm{JH}$. Considerations preliminary to the application of early and immediate loading protocols in dental implantology. Clin Oral Implants Res 2000;11:12-25.

5. Gallucci GO, Morton D, Weber HP. Loading protocols for dental implants in edentulous patients. Int $J$ Oral Maxillofac Implants 2009;24(Suppl.):132-146.

6. Ganeles J, Wismeijer D. Early and immediately restored and loaded dental implants for single-tooth and partial-arch applications. Int J Oral Maxillofac Implants 2004;19(Suppl.):92-102.

7. Morton D, Jaffin R, Weber HP. Immediate restoration and loading of dental implants: Clinical considerations and protocols. Int J Oral Maxillofac Implants 2004;19 (Suppl.):103-108.

8. Andersen E, Haanaes HR, Knutsen BM. Immediate loading of single-tooth ITI implants in the anterior maxilla: A prospective 5-year pilot study. Clin Oral Implants Res 2002;13:281-287.

9. Ericsson I, Nilson H, Lindh T, Nilner K, Randow K. Immediate functional loading of Brånemark single tooth implants. An 18 months' clinical pilot follow-up study. Clin Oral Implants Res 2000;11:26-33.

10. De Rouck T, Collys K, Cosyn J. Single-tooth replacement in the anterior maxilla by means of immediate implantation and provisionalization: A review. Int $J$ Oral Maxillofac Implants 2008;23:897-904.

11. Esposito M, Hirsch J, Lekholm U, Thomsen P. Differential diagnosis and treatment strategies for biologic complications and failing oral implants: A review of the literature. Int J Oral Maxillofac Implants 1999;14:473490.

12. Oh TJ, Yoon J, Misch CE, Wang HL. The causes of early implant bone loss: Myth or science? J Periodontol 2002;73:322-333. 
13. Frost HM. Wolff's Law and bone's structural adaptations to mechanical usage: An overview for clinicians. Angle Orthod 1994;64:175-188.

14. Isidor F. Influence of forces on peri-implant bone. Clin Oral Implants Res 2006;17(Suppl. 2):8-18.

15. Isidor F. Histological evaluation of peri-implant bone at implants subjected to occlusal overload or plaque accumulation. Clin Oral Implants Res 1997;8:1-9.

16. Miyata T, Kobayashi Y, Araki H, Ohto T, Shin K. The influence of controlled occlusal overload on periimplant tissue. Part 3: A histologic study in monkeys. Int J Oral Maxillofac Implants 2000;15:425-431.

17. Miyata T, Kobayashi Y, Araki H, Ohto T, Shin K. The influence of controlled occlusal overload on periimplant tissue. Part 4: A histologic study in monkeys. Int J Oral Maxillofac Implants 2002;17:384-390.

18. Liberati A, Altman DG, Tetzlaff J, et al. The PRISMA statement for reporting systematic reviews and metaanalyses of studies that evaluate health care interventions: Explanation and elaboration. Ann Intern Med 2009;151:W65-W94.

19. The Cochrane Collaboration. The Cochrane Handbook for systematic reviews of interventions. Available at http://handbook.cochrane.org/. Accessed December 12, 2012.

20. Schulz KF, Altman DG, Moher D; CONSORT Group. CONSORT 2010 statement: Updated guidelines for reporting parallel group randomized trials. Ann Intern Med 2010;152:726-732.

21. Degidi M, Piattelli A. Comparative analysis study of 702 dental implants subjected to immediate functional loading and immediate nonfunctional loading to traditional healing periods with a follow-up of up to 24 months. Int J Oral Maxillofac Implants 2005;20:99107.

22. Degidi M, Nardi D, Piattelli A. Immediate versus onestage restoration of small-diameter implants for a single missing maxillary lateral incisor: A 3-year randomized clinical trial. J Periodontol 2009;80: 1393-1398.

23. Güncü MB, Aslan Y, Tümer C, Güncü GN, Uysal S. Inpatient comparison of immediate and conventional loaded implants in mandibular molar sites within 12 months. Clin Oral Implants Res 2008;19:335-341.

24. Hall JA, Payne AG, Purton DG, Torr B. A randomized controlled clinical trial of conventional and immediately loaded tapered implants with screw-retained crowns. Int J Prosthodont 2006;19:17-19.

25. Ryser MR, Block MS, Mercante DE. Correlation of papilla to crestal bone levels around single tooth implants in immediate or delayed crown protocols. J Oral Maxillofac Surg 2005;63:1184-1195.

26. Schincaglia GP, Marzola R, Giovanni GF, Chiara CS, Scotti R. Replacement of mandibular molars with single-unit restorations supported by wide-body implants: Immediate versus delayed loading. A randomized controlled study. Int $J$ Oral Maxillofac Implants 2008;23:474-480.

27. Galli F, Capelli M, Zuffetti F, Testori T, Esposito M. Immediate non-occlusal vs. early loading of dental implants in partially edentulous patients: A multicentre randomized clinical trial. Peri-implant bone and softtissue levels. Clin Oral Implants Res 2008; 19:546-552.

28. Ganeles J, Zöllner A, Jackowski J, ten Bruggenkate C, Beagle J, Guerra F. Immediate and early loading of Straumann implants with a chemically modified surface (SLActive) in the posterior mandible and maxilla:
1 -year results from a prospective multicenter study. Clin Oral Implants Res 2008;19:1119-1128.

29. Turkyilmaz I, Avci M, Kuran S, Ozbek EN. A 4-year prospective clinical and radiological study of maxillary dental implants supporting single-tooth crowns using early and delayed loading protocols. Clin Implant Dent Relat Res 2007;9:222-227.

30. Crespi R, Capparé P, Gherlone E, Romanos GE. Immediate versus delayed loading of dental implants placed in fresh extraction sockets in the maxillary esthetic zone: A clinical comparative study. Int $J$ Oral Maxillofac Implants 2008;23:753-758.

31. Prosper L, Crespi R, Valenti E, Capparé P, Gherlone E. Five-year follow-up of wide-diameter implants placed in fresh molar extraction sockets in the mandible: Immediate versus delayed loading. Int J Oral Maxillofac Implants 2010;25:607-612.

32. Cannizzaro G, Leone M, Consolo U, Ferri V, Esposito M. Immediate functional loading of implants placed with flapless surgery versus conventional implants in partially edentulous patients: A 3-year randomized controlled clinical trial. Int J Oral Maxillofac Implants 2008; 23:867-875.

33. Degidi M, Piattelli A, Carinci F. Parallel screw cylinder implants: Comparative analysis between immediate loading and two-stage healing of 1,005 dental implants with a 2-year follow up. Clin Implant Dent Relat Res 2006;8:151-160.

34. Laviv A, Levin L, Usiel Y, Schwartz-Arad D. Survival of immediately provisionalized dental implants: A casecontrol study with up to 5 years follow-up. Clin Implant Dent Relat Res 2010;12(Suppl. 1):e23-e27.

35. Merli M, Bernardelli F, Esposito M. Immediate versus early nonocclusal loading of dental implants placed with a flapless procedure in partially edentulous patients: Preliminary results from a randomized controlled clinical trial. Int J Periodontics Restorative Dent 2008; 28:453-459.

36. Siebers D, Gehrke P, Schliephake H. Delayed function of dental implants: A 1 - to 7-year follow-up study of 222 implants. Int J Oral Maxillofac Implants 2010;25: 1195-1202.

37. Testori T, Bianchi F, Del Fabbro M, Szmukler-Moncler S, Francetti L, Weinstein RL. Immediate non-occlusal loading vs. early loading in partially edentulous patients. Pract Proced Aesthet Dent 2003;15:787-794, quiz 796.

38. Degidi M, lezzi G, Perrotti V, Piattelli A. Comparative analysis of immediate functional loading and immediate nonfunctional loading to traditional healing periods: A 5-year follow-up of 550 dental implants. Clin Implant Dent Relat Res 2009;11:257-266.

39. Testori T, Galli F, Capelli M, Zuffetti F, Esposito M. Immediate nonocclusal versus early loading of dental implants in partially edentulous patients: 1 -year results from a multicenter, randomized controlled clinical trial. Int J Oral Maxillofac Implants 2007;22:815-822.

40. Schwartz-Arad D, Yaniv Y, Levin L, Kaffe I. A radiographic evaluation of cervical bone loss associated with immediate and delayed implants placed for fixed restorations in edentulous jaws. J Periodontol 2004; 75:652-657.

41. Cannizzaro G, Leone M. Restoration of partially edentulous patients using dental implants with a microtextured surface: A prospective comparison of delayed and immediate full occlusal loading. Int $J$ Oral Maxillofac Implants 2003;18:512-522. 
42. Misch CE, Degidi M. Five-year prospective study of immediate/early loading of fixed prostheses in completely edentulous jaws with a bone quality-based implant system. Clin Implant Dent Relat Res 2003;5:17-28.

43. Romanos GE, Nentwig GH. Immediate versus delayed functional loading of implants in the posterior mandible: A 2 -year prospective clinical study of 12 consecutive cases. Int J Periodontics Restorative Dent 2006;26:459-469.

44. Zöllner A, Ganeles J, Korostoff J, Guerra F, Krafft T, Brägger U. Immediate and early non-occlusal loading of Straumann implants with a chemically modified surface (SLActive) in the posterior mandible and maxilla: Interim results from a prospective multicenter randomized-controlled study. Clin Oral Implants Res 2008; 19:442-450.

45. den Hartog L, Raghoebar GM, Stellingsma K, Vissink A, Meijer HJ. Immediate non-occlusal loading of single implants in the aesthetic zone: A randomized clinical trial. J Clin Periodontol 2011;38:186-194.

46. Payne AG, Tawse-Smith A, Duncan WD, Kumara R. Conventional and early loading of unsplinted ITI implants supporting mandibular overdentures. Clin Oral Implants Res 2002;13:603-609.

47. Turkyilmaz I, Tumer C. Early versus late loading of unsplinted TiUnite surface implants supporting mandibular overdentures: A 2-year report from a prospective study. J Oral Rehabil 2007;34:773-780.

48. Trisi P, Todisco M, Consolo U, Travaglini D. High versus low implant insertion torque: A histologic, histomorphometric, and biomechanical study in the sheep mandible. Int J Oral Maxillofac Implants 2011;26:837-849.

49. Ottoni JM, Oliveira ZF, Mansini R, Cabral AM. Correlation between placement torque and survival of singletooth implants. Int J Oral Maxillofac Implants 2005;20: 769-776.

50. Norton MR. The influence of insertion torque on the survival of immediately placed and restored singletooth implants. Int J Oral Maxillofac Implants 2011;26: 1333-1343.

51. Alghamdi H, Anand PS, Anil S. Undersized implant site preparation to enhance primary implant stability in poor bone density: A prospective clinical study. J Oral Maxillofac Surg 2011;69:e506-e512.

52. Rubin J, Rubin C, Jacobs CR. Molecular pathways mediating mechanical signaling in bone. Gene 2006; 367:1-16.
53. Esaki D, Matsushita Y, Ayukawa Y, Sakai N, Sawae Y, Koyano K. Relationship between magnitude of immediate loading and peri-implant osteogenesis in dogs. Clin Oral Implants Res 2011; 23:1290-1296.

54. Piattelli A, Corigliano M, Scarano A, Costigliola G, Paolantonio $M$. Immediate loading of titanium plasmasprayed implants: An histologic analysis in monkeys. J Periodontol 1998;69:321-327.

55. Romanos GE, Toh CG, Siar CH, Swaminathan D. Histologic and histomorphometric evaluation of periimplant bone subjected to immediate loading: An experimental study with Macaca fascicularis. Int J Oral Maxillofac Implants 2002;17:44-51.

56. Neugebauer J, Traini T, Thams U, Piattelli A, Zöller JE. Peri-implant bone organization under immediate loading state. Circularly polarized light analyses: a minipig study. J Periodontol 2006;77:152-160.

57. De Kok IJ, Chang SS, Moriarty JD, Cooper LF. A retrospective analysis of peri-implant tissue responses at immediate load/provisionalized microthreaded implants. Int J Oral Maxillofac Implants 2006;21:405412.

58. Kan JY, Rungcharassaeng K, Lozada JL, Zimmerman G. Facial gingival tissue stability following immediate placement and provisionalization of maxillary anterior single implants: A 2- to 8-year follow-up. Int $J$ Oral Maxillofac Implants 2011;26:179-187.

59. Cosyn J, Eghbali A, De Bruyn H, Collys K, Cleymaet R, De Rouck T. Immediate single-tooth implants in the anterior maxilla: 3-year results of a case series on hard and soft tissue response and aesthetics. J Clin Periodontol 2011;38:746-753.

60. Tortamano P, Camargo LO, Bello-Silva MS, Kanashiro LH. Immediate implant placement and restoration in the esthetic zone: A prospective study with 18 months of follow-up. Int J Oral Maxillofac Implants 2010;25: 345-350.

Correspondence: Dr. Hom-Lay Wang, Department of Periodontics and Oral Medicine, University of Michigan School of Dentistry, 1011 N. University Ave., Ann Arbor, MI 48109-1078. Fax: 734/936-0374; e-mail: homlay@ umich.edu.

Submitted February 7, 2012; accepted for publication March 23, 2012 